\title{
Why data on frailty and SARS-CoV-2 infection are basic to progress
}

\author{
A. Zucchelli ${ }^{1} \cdot$ E. Bologna ${ }^{2} \cdot$ A. Marengoni ${ }^{3}[$ \\ Received: 1 January 2021 / Accepted: 23 March 2021 / Published online: 2 April 2021 \\ (c) The Author(s), under exclusive licence to Springer Nature Switzerland AG 2021
}

\begin{abstract}
Several studies showed that frailty was a predictor of in-hospital death in older adults with COVID-19. The mechanisms through which frailty increases the severity of COVID-19 are several, including immunosenescense and dysregulated inflammation. Whether individuals affected by frailty exhibit a higher susceptibility to SARS-CoV-2 infection remains an open question. Here we report the case series of 40 older persons that in February 2020, before the first case of COVID-19 was detected in Italy, went together on a winter holiday. Back home, 7 of them developed influenza-like symptoms and one was hospitalized due to COVID-19 pneumonia. Between May and July, the seniors were offered the possibility to be tested for SARS-CoV-2 antibody positivity. Twenty-seven of them accepted: 13 had a positive serological test whereas no active infection was found. Comparing the characteristics of those who tested positive and the others, we found that the former group was frailer, exhibiting higher Clinical Frailty Scale scores.
\end{abstract}

Keywords Frailty $\cdot$ COVID-19 $\cdot$ SARS-CoV-2 $\cdot$ Older persons

The first studies on risk factors associated to adverse outcomes from coronavirus disease 2019 (COVID-19) showed that chronological age and pre-existing chronic diseases were important determinants of mortality. Onder et al. found that patients with COVID-19 who died in March in Italy had a mean age of 79.5 years and a mean number of preexisting diseases of 2.7 [1]. Soon after, Bellelli et al. showed that frailty was a strong predictor of in-hospital death or intensive care unit admission in a sample of adults admitted for COVID-19 in Northern Italy [2]. This study was followed by a number of other reports from different countries, such as England [3], Sweden [4], and China [5], showing very similar findings. Furthermore, frailty, measured years before and irrespectively of the tool used for its assessment, appears to be able to identify those persons at higher risk of severe COVID-19 [6]. Recently, we showed that frailty not only was independently associated with mortality in patients affected by COVID-19, but also added prognostic

A. Zucchelli

a.zucchelli001@unibs.it

1 Department of Information Engineering, Università degli Studi di Brescia, vle Branze 38, 25123 Brescia, Italy

2 Fondazione Piera, Pietro e Giovanni Ferrero, Alba, Italy

3 Department of Clinical and Experimental Sciences, Università degli Studi di Brescia, Brescia, Italy information beyond chronological age in those aged 70 years or older [7]. Beside its role in COVID-19, frailty has been shown to be such a good predictor of adverse events in the general population [8] that its evaluation has been implemented in primary care in the United Kingdom, as a tool aiming at stratifying older persons' risk of developing poor outcomes $[9,10]$.

\section{What is frailty and why it is linked with COVID-19}

Frailty is a multisystemic syndrome characterized by decreased physiological and functional reserve: the biological changes of ageing are widespread to most tissues and organs, acting as the main pathogenic mechanism for frailty itself [11]. The multiorgan involvement found in frailty leads to the dysregulation of the neuromuscular, endocrine, and immune systems: the normal adaptation to events is compromised and the ability to cope with acute stressors is weakened. Even though frailty often coexist with multimorbidity and/or functional impairment in older adults, there is increasing consensus that frailty is a unique clinical entity and, as such, people aged 70 year or older should be screened for the presence of frailty; however, this is rarely the case. 
The mechanisms through which frailty may increase the severity of COVID-19 are several. The age-related decline in immune function can contribute to frailty and in turn to an increased susceptibility to acute infectious diseases. Previously, frailty has been found associated with lower probability of recovery in older persons hospitalized with influenza and acute respiratory illness [12]. In particular, changes in the innate immunity could enhance a pro-inflammatory state which is a principal component of frailty. In a systematic review of the literature, frailty and pre-frailty were associated with higher levels of circulating inflammatory cytokines [13]. The renin-angiotensin system is also hypothesized to play an important role in the pathogenesis of frailty through a shift in the balance between the proinflammatory vs. antiinflammatory action of Ang II type 1- and Ang II type 2 receptor, respectively, resulting in increased inflammation, oxidative stress, and apoptosis [14]. Thus, in COVID-19, frailty could be a marker of a substrate sensitive to infections, leaving as open question if it could also enhance a dysregulated inflammatory response. Further, frailty has a strong relationship with sarcopenia and malnutrition which have been associated with poorer outcomes in adult affected by COVID-19 [15].

Not less relevant, frailty is particularly present in economic disadvantaged and social isolated older persons which are hardly hit by COVID-19 consequences [16].

\section{Frailty and susceptibility to SARS-CoV-2 infection}

Whether individuals affected by frailty exhibit a higher susceptibility to SARS-CoV-2 infection remains an open question. Here we report the case series of 40 older persons that in February 2020, 2 weeks before the first case of COVID-19 was detected in Italy, went together on a winter holiday in Liguria, North-Western Italy. The event was organized by the Ferrero Foundation, a no-profit organization aiming at promoting healthy and active aging for the retired seniors who worked for the Ferrero Company. Back home, seven of them developed influenza-like symptoms and one was hospitalized due to COVID-19 pneumonia. At that time, due to shortage in tests and resources, only the hospitalized person and his husband (who always remained asymptomatic) were tested for SARS-CoV-2. Between the 5th of May and the 6th of July, the Ferrero Foundation offered to all the seniors who were together on vacation, the possibility to be tested for SARS-CoV-2 antibody positivity (lateral flow immunoassay-LFIA serological tests). A nasopharyngeal swab to check any current infection was also proposed. Twenty seven of them accepted: $13 \mathrm{had}$ a positive serological test whereas no active infection was found. Comparing the demographical and clinical characteristics of those who tested positive and the others, we found that the former group was frailer, exhibiting higher Clinical Frailty Scale (CFS) scores [17] (Table 1). The CFS is an easy-to-perform frailty assessment tool that ranges between one (no frailty) and nine (terminally ill). It has been shown to have an accuracy similar to those of comprehensive and validated multidimensional frailty scoring system (such as the frailty index) [17]. For the presented data, the CFS was assessed by a trained nurse, based on the information and clinical status of the participants before the winter holiday. No other differences between the two groups were detected. Due to the very limited number of persons analysed and the uncertainty about the life interval of SARS-CoV-2 antibodies, we cannot trace any formal conclusion. The absence of SARS-CoV-2 antibodies does not exclude the possibility that a participant was infected but the virus did not elicit any immune response, as already reported in the literature [18]. The presence of antibodies does not exclude that participants were infected after the winter holiday, although this possibility is remote considering the lockdown that was established in Italy during the first wave since the beginning of March and the strong advice to older persons to remain at home and avoid social contacts. However, we can surely suggest some fields of future research and eventually action.

\section{Future research}

Frailty has already been associated with increased risk of infection. A higher probability of fungal, bacterial and viral (such as cytomegalovirus [19]) infection has been found in older persons affected by frailty [20, 21]. Furthermore, frailty has been associated with increased risk of post-operative infection in hip fracture [22] and elective non-cardiac surgery [23]. Immunosenescence, impairment of cutaneous and mucosal barriers, the presence of comorbidities as well as micronutrient and protein deficiencies has been proposed as possible mechanisms of the increased risk of infection in frail persons [20].

If future studies confirm that persons affected by frailty have higher chances of being infected by SARS-CoV-2, targeted preventive approaches based on frailty assessment might be carried through.

Personalized approaches are warranted as it might be possible, for example, that the effectiveness of a SARS-CoV-2 vaccine varies with frailty, as already shown for influenza [24]: studies investigating the protective answer elicited by a vaccine in the frailer strata of the population should be conducted, to allow the construction of ad hoc vaccination protocols for persons affected by frailty. Recently, the safety and efficacy of the ChAdOx $1 \mathrm{nCoV}-19$ vaccine in older persons were tested, but those with a Clinical Frailty Score (CFS) of 4 or higher were excluded [25]. 
Table 1 Characteristics of "Ferrero's Senior" participating to the SARS-CoV-2 infection screening procedure, stratified by serological test result

\begin{tabular}{|c|c|c|c|c|}
\hline & Overall $(N=27)$ & $\begin{array}{l}\text { Negative serological test } \\
(N=14-51.9 \%)\end{array}$ & $\begin{array}{l}\text { Positive serological test } \\
(N=13-48.1 \%)\end{array}$ & $p$ \\
\hline Age [Mean (SD)] & $77.59(5.61)$ & $77.00(6.78)$ & $78.23(4.19)$ & 0.579 \\
\hline Male gender (\%) & $11(40.7)$ & $5(35.7)$ & $6(46.2)$ & 0.704 \\
\hline Widow/Widower (\%) & $7(28.0)$ & $4(33.3)$ & $3(23.1)$ & 0.673 \\
\hline Smoking (previous or actual) $(\%)$ & $9(33.3)$ & $4(28.6)$ & $5(38.5)$ & 0.695 \\
\hline Total number of drugs prescribed [median (IQR)] & $3.00[2.00,5.50]$ & $3.50[2.00,5.50]$ & $3.00[2.00,5.00]$ & 0.941 \\
\hline Hypertension (\%) & $16(59.3)$ & $10(71.4)$ & $6(46.2)$ & 0.252 \\
\hline Obesity (\%) & $3(11.1)$ & $1(7.1)$ & $2(15.4)$ & 0.596 \\
\hline Ischemic heart disease $(\%)$ & $2(7.4)$ & $1(7.1)$ & $1(7.7)$ & 1.000 \\
\hline Heart failure $(\%)$ & $1(3.7)$ & $0(0.0)$ & $1(7.7)$ & 0.481 \\
\hline COPD $(\%)$ & $1(3.7)$ & $1(7.1)$ & $0(0.0)$ & 1.000 \\
\hline Cognitive decline $(\%)$ & $3(11.1)$ & $3(21.4)$ & $0(0.0)$ & 0.222 \\
\hline Depression $(\%)$ & $2(7.4)$ & $1(7.1)$ & $1(7.7)$ & 1.000 \\
\hline $2+$ Chronic conditions $(\%)$ & $9(33.3)$ & $5(35.7)$ & $4(30.8)$ & 1.000 \\
\hline Angiotensin receptor blockers or ace-inhibitors (\%) & $11(40.7)$ & $6(42.9)$ & $5(38.5)$ & 1.000 \\
\hline Statins $(\%)$ & $10(37.0)$ & $5(35.7)$ & $5(38.5)$ & 1.000 \\
\hline Metformin (\%) & $2(7.4)$ & $0(0.0)$ & $2(15.4)$ & 0.222 \\
\hline Any neuropsychotropic drug & $9(33.3)$ & $5(35.7)$ & $4(30.8)$ & 1.000 \\
\hline $\begin{array}{l}\text { Participating to the physical activity programme of Fer- } \\
\text { rero foundation }(\%)\end{array}$ & $20(74.1)$ & $10(71.4)$ & $10(76.9)$ & 1.000 \\
\hline Clinical Frailty Scale Score [Median (IQR)] & $3.00[2.00,3.00]$ & $2.00[1.25,2.75]$ & $3.00[3.00,3.00]$ & 0.015 \\
\hline
\end{tabular}

Any neuropsychotropic drug = any drug chronically prescribed among serotonin selective receptor inhibitors (SSRI), serotonin noradrenalin receptor inhibitors (SNRI), trazodone, benzodiazepines, antiepileptics, and opioids

$S D$ standard deviation, $I Q R$ interquartile range, $C O P D$ chronic obstructive pulmonary disease

Furthermore, due to the strong association between frailty and disability, persons with frailty have often contacts with several caregivers, both formal and informal. It is likely that preventive strategies should also reach those who care for frail individuals: the lack of staff testing and protective equipment in nursing homes has proven to have extremely severe consequences in terms of morbidity and mortality [26].

Lastly, COVID-19 may manifest itself with aspecific signs and symptoms in older persons affected by frailty [27]. Routine evaluation of frailty, both in primary care and hospital settings, would help to identify those persons who might benefit from a global and careful evaluation to be correctly diagnosed.

In conclusion, the evaluation of frailty may be helpful in research as well as in clinical practice. Studies evaluating the incidence of SARS-CoV-2 infection according to the presence of frailty in the general population may advance progress in the understanding of COVID-19 spread and pathogenesis, whereas frailty systematic assessment in primary care may help to prompt the recognition of some well-known contributors to frailty development, such as undernutrition, physical inactivity, polypharmacy, and social isolation.

Acknowledgement We would like to deeply thank Laura Rolfo and Fabio Timelli for their invaluable support in taking care of "our Seniors".

\section{Declarations}

Conflict of interest The authors declare they have no conflict of interest.

Human and animal rights The present research was conducted in accordance with the ethical standarrds as laid down in the 1964 Declaration of Helsinki and its later amendments.

Informed consent All study participants signed an informed consent about data utilization for scientific research purposes and dissemination upon enrollement to the activities offered by Fondazione Piera, Pietro e Giovanni Ferrero. 


\section{References}

1. Onder G, Rezza G, Brusaferro S (2020) Case-fatality rate and characteristics of patients dying in relation to COVID-19 in Italy. JAMA. https://doi.org/10.1001/jama.2020.4683

2. Bellelli G, Rebora P, Citerio G (2020) The role of frailty in COVID-19 patients. Intensive Care Med 46:1958

3. Hewitt J, Carter B, Vilches-Moraga A et al (2020) The effect of frailty on survival in patients with COVID-19 (COPE): a multicentre, European, observational cohort study. Lancet Public Health 5:1555-1559

4. Hägg S, Jylhävä J, Wang Y et al (2020) Age, frailty, and comorbidity as prognostic factors for short-term outcomes in patients with coronavirus disease 2019 in geriatric care. J Am Med Dir Assoc 21:1555-1559

5. Ma Y, Hou L, Yang X et al (2020) The association between frailty and severe disease among COVID-19 patients aged over 60 years in China: a prospective cohort study. BMC Med. https://doi.org/ 10.1186/s12916-020-01761-0

6. Petermann-Rocha F, Hanlon P, Gray S et al (2020) Comparison of two different frailty measurements and risk of hospitalisation or death from COVID-19: findings from UK Biobank. BMC Med. https://doi.org/10.1186/s12916-020-01822-4

7. Marengoni A, Zucchelli A, Vetrano D et al (2020) Beyond chronological age: Frailty and multimorbidity predict in-hospital mortality in patients with coronavirus disease. J Gerontol Ser A. https:// doi.org/10.1093/gerona/glaa291

8. Zucchelli A, Vetrano DL, Grande G et al (2019) Comparing the prognostic value of geriatric health indicators: a population-based study. BMC Med 17:185. https://doi.org/10.1186/ s12916-019-1418-2

9. Clegg A, Bates C, Young J et al (2016) Development and validation of an electronic frailty index using routine primary care electronic health record data. Age Ageing 45:353-360

10. National Health System (2020) Electronic Frailty Index. https:// www.england.nhs.uk/ourwork/clinical-policy/older-people/frail ty/efi/. Accessed 19 Nov 2020

11. Thillainadesan J, Scott I, Le Couteur D (2020) Frailty, a multisystem ageing syndrome. Age Ageing 49:758-763

12. Lees C, Godin J, McElhaney JE et al (2020) Frailty hinders recovery from influenza and acute respiratory illness in older adults. $\mathbf{J}$ Infect Dis. https://doi.org/10.1016/j.arr.2016.08.006

13. Soysal P, Stubbs B, Lucato P et al (2016) Inflammation and frailty in the elderly: a systematic review and meta-analysis. Ageing Res Rev 31:1-8

14. Abadir P (2011) The frail renin-angiotensin system. Clin Geriatr Med 27:53-65

15. Laviano A, Koverech A, Zanett IM (2020) Nutrition support in the time of SARS-CoV-2 (COVID-19). Nutrition 74:110834
16. Calderón-Larrañaga A, Dekhtyar S, Vetrano D et al (2020) COVID-19: risk accumulation among biologically and socially vulnerable older populations. Ageing Res Rev 63:101149

17. Rockwood K, Song X, MacKnight C et al (2005) A global clinical measure of fitness and frailty in elderly people. Canad Med Assoc J 173:489-495

18. Chen Y, Zuiani A, Fischinger S et al (2020) Quick COVID-19 healers sustain anti-SARS-CoV-2 antibody production. Cell 183:1496-1507

19. Schmaltz HN, Fried LP, Xue QL et al (2005) Chronic cytomegalovirus infection and inflammation are associated with prevalent frailty in community-dwelling older women. J Am Geriatr Soc 53:747-754

20. Tannou T, Koeberle S, Manckoundia P et al (2019) Multifactorial immunodeficiency in frail elderly patients: contributing factors and management. Med Mal Infect 49:167-172

21. Htwe TH, Mushtaq A, Robinson SB et al (2007) Infection in the elderly. Infect Dis Clin N Am 21:711-743

22. Chen CL, Chen CM, Wang CY et al (2019) Frailty is associated with an increased risk of major adverse outcomes in elderly patients following surgical treatment of hip fracture. Sci Rep 9:19135

23. Birkelbach O, Morgeli R, Spies C et al (2019) Routine frailty assessment predicts postoperative complications in elderly patients across surgical disciplines: a retrospective observational study. BMC Anesthesiol 19:204

24. Andrew M, Shinde V, Ye L et al (2017) The importance of frailty in the assessment of influenza vaccine effectiveness against influenza-related hospitalization in elderly people. J Infect Dis 216:405-414

25. Ramasamy M, Minassian A, Ewer K et al (2020) Safety and immunogenicity of ChAdOx $1 \mathrm{nCoV}-19$ vaccine administered in a prime-boost regimen in young and old adults (COV002): a single-blind, randomised, controlled, phase $2 / 3$ trial. Lancet 396:1979-1993

26. American Geriatric Society (2020) American Geriatrics Society Policy Brief: COVID-19 and Nursing Homes. J Am Geriatr Soc 68:908-911

27. Poco P, Aliberti M, Dias M et al (2020) Divergent: age, frailty, and atypical presentations of COVID-19 in hospitalized patients. J Gerontol Ser A. https://doi.org/10.1093/gerona/glaa280

Publisher's Note Springer Nature remains neutral with regard to jurisdictional claims in published maps and institutional affiliations. 